\title{
Hyperglycemia and Renal Mass Ablation Synergistically Augment Albuminuria in the Diabetic Subtotally Nephrectomized Rat: Implications for Modeling Diabetic Nephropathy
}

\author{
Li-Hao Chen Bailey Stead Suzanne L. Advani Noreen Yaqoob Kerri Thai \\ M. Golam Kabir Darren A. Yuen Kim A. Connelly Richard E. Gilbert \\ Andrew Advani
}

Keenan Research Centre of the Li Ka Shing Knowledge Institute, St. Michael's Hospital, Toronto, Ont., Canada

\section{Key Words}

Diabetic nephropathy $\cdot$ Subtotal nephrectomy $\cdot$ Streptozotocin $\cdot$ Albuminuria $\cdot$

Glomerular hypertrophy $\cdot$ Nephrin $\cdot$ Glomerulosclerosis $\cdot$ Experimental model

\begin{abstract}
Background/Aims: While experimental models that emulate diabetic nephropathy are valuable tools for elucidating pathogenetic mechanisms and developing novel therapies, existing models imperfectly recapitulate human disease. In diabetes, hyperglycemia and hemodynamic forces act in concert to induce renal injury. Accordingly, in the present study, we combined streptozotocin-induced diabetes with surgical ablation of 5/6 of the kidney mass with the aim of evaluating their additive effects on renal function and glomerular morphology. Methods: Female F344 rats were randomized to undergo subtotal nephrectomy (SNx) either at baseline or following 4 weeks of diabetes. Results: In comparison to sham rats, rats with diabetes or rats after SNx surgery, diabetic subtotally nephrectomized (DM-SNx) rats demonstrated an increase in systolic blood pressure, glomerular volume and mesangial matrix. Albuminuria was synergistically increased by hyperglycemia and renal mass ablation associated with decreased nephrin expression. In contrast, glomerular capillary rarefaction and glomerular filtration rate were similarly reduced in SNx and DM-SNx rats. Conclusion: The DM-SNx rat recapitulates some of the features of human disease, most notably augmented albuminuria. Since this model avoids the deletion or overexpression of gene(s) linked to the pathogenesis of nephropathy, the DM-SNx rat model represents a complementary tool for the trial of novel therapies.
\end{abstract}

Copyright $\odot 2012$ S. Karger AG, Basel 


\section{Introduction}

One of the major limitations to the study of pathogenetic mechanisms in diabetic nephropathy is the lack of a rodent model that reproducibly develops renal disease analogous to that seen in human patients. In recent years, technological advances in genetic manipulation have enabled the generation of promising experimental models lacking in (or overexpressing) genes inextricably linked to the progression of the condition [1]. While undoubtedly an advance, such models possess limitations when it comes to their use in the development and testing of novel therapies. Although deletion of a particular gene may accelerate renal injury in diabetes, its absence may also render a promising experimental therapy ineffective if such a therapy's mechanism of action is reliant on the affected pathway [2].

Hypertension is present in approximately $70 \%$ of individuals with diabetes [3] and is an independent predictor of the future risk of both the development and progression of renal disease [4]. Such epidemiological evidence, together with the well-documented beneficial effects of anti-hypertensive therapy $[5,6]$, pays testament to the synergistic relationship between hyperglycemia and hemodynamic forces in mediating renal decline. One well-described and well-established means of inducing renal injury as a consequence of hemodynamic stress is the surgical ablation of $5 / 6$ of the renal mass (subtotal nephrectomy, SNx). Although landmark studies of ACE inhibition testify to this model's pedigree in pharmaceutical development [7], the absence of hyperglycemia limits its applicability to the diabetic setting. In this context, we sought to develop a novel rodent model of renal disease by performing SNx surgery in rats with pre-existing streptozotocin (STZ)-induced diabetes.

\section{Methods}

\section{Animals}

Female Fischer (F344) rats aged 8 weeks were randomized to undergo either SNx or sham surgery ( $\mathrm{n}=8-12$ /group) as previously described [8]. Following an 8 -hour fast, diabetes was induced with a single intraperitoneal injection of STZ $(45 \mathrm{mg} / \mathrm{kg}$ in $0.1 \mathrm{M}$ citrate buffer $\mathrm{pH} 4.5), 4$ weeks before SNx surgery, and animals were followed for 8 weeks after renal mass ablation. Blood glucose was determined weekly and 1 unit of insulin (Humulin N isophane; Eli Lilly, Toronto, Ont., Canada) was administered subcutaneously (s.c.) if blood glucose was greater than $32 \mathrm{~mm}$. Diabetic rats received 1 unit of insulin $48 \mathrm{~h}$ prior to surgery. Peri-operative fluid management consisted of the administration of Ringer's lactate solution s.c. 5-10 $\mathrm{ml}$ immediately pre-operatively, $3-4 \mathrm{ml}$ post-operatively and $5 \mathrm{ml}$ daily for the next 2 days. $\mathrm{HbA}_{1 \mathrm{c}}$ was determined using A1cNow+ (Bayer, Sunnyvale, Calif., USA). The glomerular filtration rate (GFR) was determined by FITC-inulin clearance as previously described [8]. Urine albumin excretion was determined using AssayMax Rat Albumin ELISA kit (Assaypro, St. Charles, Mo., USA). Systolic blood pressure (SBP) was measured with a 2F micromanometer (Model SPR-838; Millar Instruments, Houston, Tex., USA) and analyzed using Chart Software v5.6 (AD Instruments, Bella Vista, N.S.W., Australia). All experimental procedures adhered to the guidelines of the Canadian Council on Animal Care and were approved by the St. Michael's Hospital Animal Care Committee.

\section{Immunohistochemistry}

Immunohistochemistry was performed as previously described [8-11] with antibodies in the following concentrations: JG-12 1:1,000 (Bender MedSystems GmbH, Vienna, Austria), nephrin 1:500 (R\&D Systems, Minneapolis, Minn., USA), WT1 1:1,000 (Santa Cruz Biotechnology, Santa Cruz, Calif., USA) and collagen IV 1:100 (Southern Biotech, Birming- 
ham, Ala., USA). Kidney sections were scanned with the Aperio ScanScope system (Aperio Technologies Inc., Vista, Calif., USA) and analyzed using ImageScope (Aperio Technologies Inc.). For the determination of capillary density and nephrin, the proportional glomerular area of positive immunostaining was determined in 30 randomly selected glomerular profiles from each kidney section $[8,10]$. Glomerular nuclei positively immunostaining for WT1 were quantified in 30 glomerular profiles from each kidney section as previously described [9]. Cortical tubulointerstitial collagen IV was determined as the proportional cortical area positively immunostaining in six random non-overlapping fields $\times 100$ magnification.

\section{Glomerular Volume}

The glomerular volume was calculated on PAS-stained kidney sections (30 glomerular profiles from each rat) as described previously [11].

\section{Glomerulosclerosis Index}

The magnitude of glomerulosclerosis was calculated from at least 50 glomeruli in PASstained kidney sections using a semi-quantitative scoring system as previously described $[8,9]$.

\section{Statistics}

Data are expressed as means \pm SEM. Statistical significance was determined by one-way ANOVA with Newman-Keuls post-test. Two-way ANOVA was performed for assessing the interaction between STZ-induced diabetes and SNx. All statistical analyses were performed using GraphPad Prism 5 for Mac OS X (GraphPad Software Inc., San Diego, Calif., USA).

\section{Results}

\section{Development of the DM-SNx Model}

We anticipated that concurrent STZ-induced diabetes and SNx surgery would adversely affect mortality and therefore selected a rat sex and strain (female F344 rats) in which renal mass ablation would induce comparatively mild disease. In pilot studies, urine protein excretion in female F344 rats was approximately one-third of that seen in male Sprague Dawley animals 8 weeks after SNx surgery (urine protein excretion in $\mathrm{mg} / 24 \mathrm{~h}$ : male Sprague Dawley SNx $109.61 \times / \div 1.5$, female F344 SNx $30.93 \times / \div 1.4, \mathrm{p}<0.05$ ). To allow recovery from acute development of hyperglycemia, SNx surgeries were performed 4 weeks after diabetes induction.

\section{Renal Function in DM-SNx Rats}

Following our initial exploratory studies, four groups of rats were studied: sham-operated rats (sham), subtotally nephrectomized rats (SNx), STZ-diabetic rats (DM) and STZdiabetic rats that underwent SNx surgery (DM-SNx) (table 1). Eight weeks after sham or SNx surgery, there was no difference in glycemic control between DM and DM-SNx rats. SNx surgery resulted in an increase in SBP in non-diabetic and a larger increase in diabetic SNx rats (table 1). Whereas GFR was increased in DM rats, consistent with the hyperfiltrative phase of diabetic nephropathy, it was reduced in rats after renal mass ablation (table 1). The combination of diabetes and SNx did not cause a greater decrease in GFR than SNx alone (table 1). In contrast, while albuminuria was minimally increased in DM rats and moderately increased in SNx rats, it was markedly and synergistically augmented with the combination of diabetes and SNx in DM-SNx rats (fig. 1). 
Fig. 1. Albumin excretion rate in sham-operated, DM, SNx and DM-SNx rats. ${ }^{*} \mathrm{p}<0.001$ vs. all other groups. $\mathrm{p}<0.001$ for interaction.

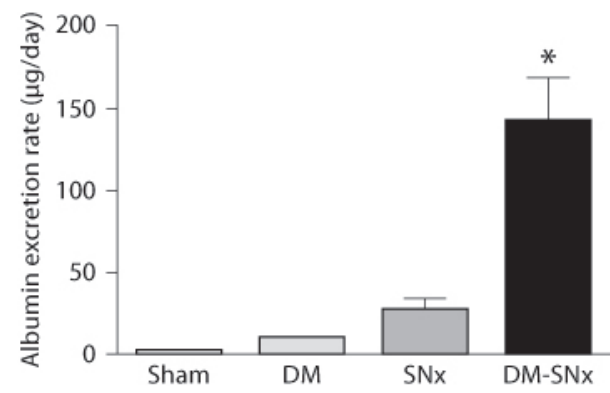

Table 1. Functional characteristics of sham-operated, DM, SNx and DM-SNx rats

\begin{tabular}{lllllll}
\hline & $\begin{array}{l}\text { Body weight } \\
\mathrm{g}\end{array}$ & $\begin{array}{l}\text { Left kidney } \\
\text { weight, } \mathrm{L}\end{array}$ & $\begin{array}{l}\text { Left kidney } \\
\text { weight:body } \\
\text { weight, } \%\end{array}$ & $\begin{array}{l}\mathrm{HbA}_{1 \mathrm{c}} \\
\%\end{array}$ & $\begin{array}{l}\text { SBP } \\
\mathrm{mm} \mathrm{Hg}\end{array}$ & $\begin{array}{l}\mathrm{GFR} \\
\mathrm{ml} / \mathrm{min} / \mathrm{kg}\end{array}$ \\
\hline Sham & $185 \pm 3$ & $0.57 \pm 0.01$ & $0.31 \pm 0.01$ & $4.5 \pm 0.1$ & $110 \pm 4$ & $5.7 \pm 0.3$ \\
DM & $131 \pm 4^{\mathrm{a}}$ & $0.60 \pm 0.03$ & $0.54 \pm 0.02^{\mathrm{a}}$ & $11.0 \pm 0.2^{\mathrm{a}}$ & $127 \pm 6$ & $7.6 \pm 0.4^{\mathrm{h}}$ \\
SNx & $171 \pm 4^{\mathrm{b}}$ & $0.63 \pm 0.04$ & $0.38 \pm 0.03^{\mathrm{b}}$ & $4.3 \pm 0.1^{\mathrm{b}}$ & $144 \pm 5^{\mathrm{f}}$ & $2.8 \pm 0.4^{\mathrm{a}, \mathrm{b}}$ \\
DM-SNx & $126 \pm 5^{\mathrm{a}, \mathrm{c}}$ & $0.75 \pm 0.03^{\mathrm{a}, \mathrm{d}, \mathrm{e}}$ & $0.60 \pm 0.03^{\mathrm{a}, \mathrm{c}}$ & $11.7 \pm 0.7^{\mathrm{a}, \mathrm{c}}$ & $166 \pm 12^{\mathrm{a}, \mathrm{d}, \mathrm{g}}$ & $3.1 \pm 0.5^{\mathrm{a}, \mathrm{b}}$ \\
\hline
\end{tabular}

${ }^{\mathrm{a}} \mathrm{p}<0.001$ vs. sham, ${ }^{\mathrm{b}} \mathrm{p}<0.001$ vs. DM, ${ }^{\mathrm{c}} \mathrm{p}<0.001$ vs. SNx,${ }^{\mathrm{d}} \mathrm{p}<0.01$ vs. DM, ${ }^{\mathrm{e}} \mathrm{p}<0.05$ vs. SNx, ${ }^{\mathrm{f}} \mathrm{p}<$ 0.01 vs. sham, ${ }^{\mathrm{g}} \mathrm{p}<0.05$ vs. $\mathrm{SNx},{ }^{\mathrm{h}} \mathrm{p}<0.05$ vs. sham.

Table 2. Glomerulosclerosis index (GSI) and the percentage of glomeruli with glomerulosclerosis in shamoperated, DM, SNx and DM-SNx rats

\begin{tabular}{|c|c|c|c|c|c|c|}
\hline & GSI & $\begin{array}{l}\text { Grade } 0 \\
0 \%\end{array}$ & $\begin{array}{l}\text { Grade } 1 \\
1-25 \%\end{array}$ & $\begin{array}{l}\text { Grade } 2 \\
26-50 \%\end{array}$ & $\begin{array}{l}\text { Grade } 3 \\
51-75 \%\end{array}$ & $\begin{array}{l}\text { Grade } 4 \\
76-100 \%\end{array}$ \\
\hline Sham & $0.34 \pm 0.04$ & $66.4 \pm 4.1$ & $33.2 \pm 4.1$ & $0.4 \pm 0.2$ & 0 & 0 \\
\hline DM & $0.96 \pm 0.09^{a}$ & $18.2 \pm 5.3$ & $67.8 \pm 4.8$ & $13.5 \pm 4.2$ & $0.4 \pm 0.3$ & 0 \\
\hline $\mathrm{SNx}$ & $1.02 \pm 0.14^{\mathrm{a}}$ & $26.6 \pm 5.6$ & $54.0 \pm 5.0$ & $13.6 \pm 3.5$ & $2.5 \pm 1.0$ & $3.5 \pm 2.0$ \\
\hline DM-SNx & $2.05 \pm 0.31^{\mathrm{b}}$ & $8.9 \pm 2.6$ & $32.8 \pm 8.7$ & $20.8 \pm 4.4$ & $19.7 \pm 4.6$ & $17.8 \pm 8.5$ \\
\hline
\end{tabular}

Glomerulosclerosis grade determined as the estimated percentage of the glomerular profile affected. ${ }^{\mathrm{a}} \mathrm{p}<0.05$ vs. sham, ${ }^{\mathrm{b}} \mathrm{p}<0.001$ vs. all other groups.

\section{Glomerulosclerosis, Glomerular Volume and Glomerular Capillary Density in \\ DM-SNx Rats}

Despite a minimal rise in albuminuria in DM rats relative to sham rats, mesangial matrix deposition was notably increased (table 2; fig. 2). However, in contrast to the segmental distribution of glomerulosclerosis observed in SNx rats, matrix deposition in DM animals was diffusely distributed throughout the glomerulus (table 2). The glomerulosclerosis index was increased in DM-SNx relative to either DM or SNx rats alone, primarily as a consequence of an increase in the number of glomeruli showing areas of moderate or severe sclerosis (table 2). The glomerular volume was increased after SNx surgery and was augmented in the 
Fig. 2. Representative periodic acid-Schiff-stained kidney sections from sham-operated (a), $\mathrm{DM}$ (b), $\mathrm{SNx}$ (c) and DM-SNx (d) rats. Original magnification $\times 400$.

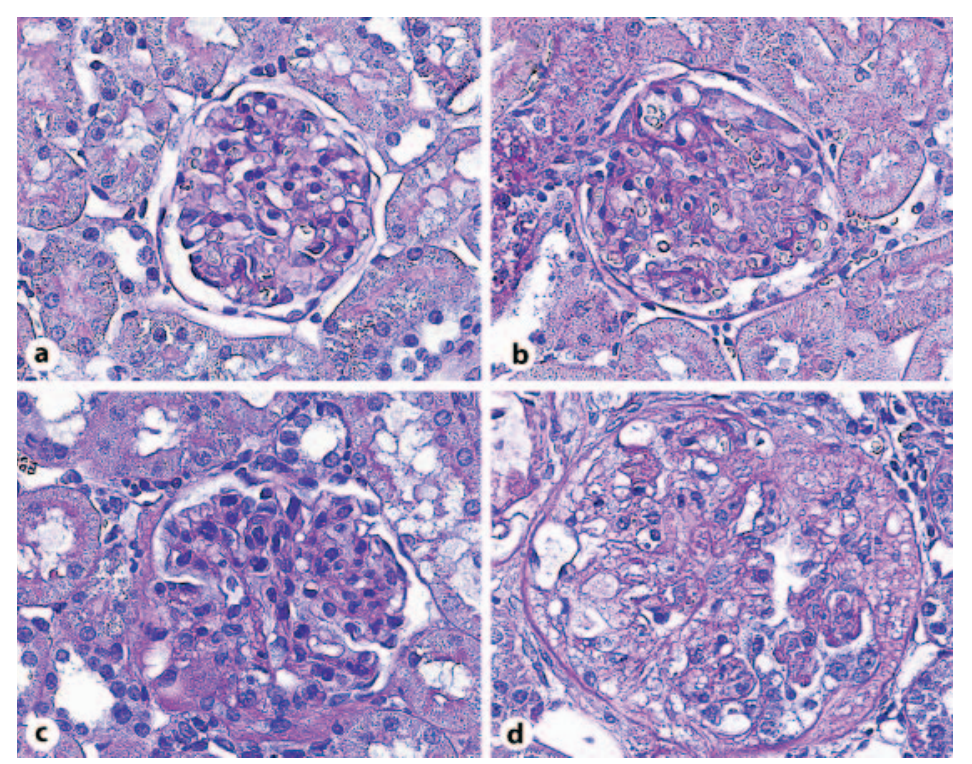

Fig. 3. Glomerular volume in sham-operated, DM, SNx and DM-SNx rats. * $\mathrm{p}<0.001$ vs. sham, ${ }^{\dagger} \mathrm{p}<0.01$ vs. DM, ${ }^{\ddagger} \mathrm{p}<$ 0.001 vs. DM, ${ }^{\S} \mathrm{p}<0.05$ vs. SNx.

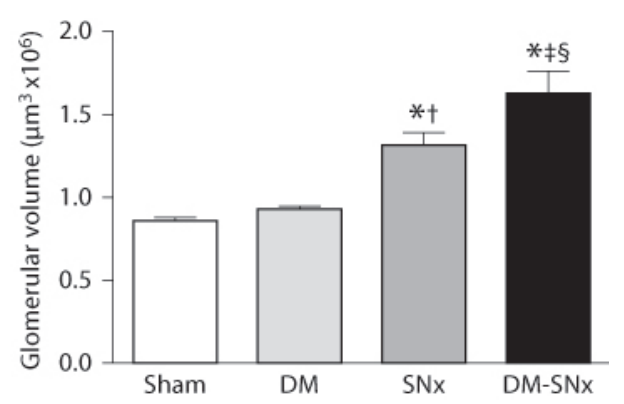

setting of diabetes (i.e. in DM-SNx rats) (fig. 3). Although the glomerular volume was increased, consistent with the changes in GFR, the glomerular capillary density was reduced in both SNx and DM-SNx rats (fig. 4). In contrast to glomerular changes in DM-SNx rats, tubulointerstitial fibrosis, as determined by collagen IV immunostaining, was not increased (proportional area of cortical tubulointerstitial collagen IV (AU): sham $0.077 \pm 0.007, \mathrm{SNx}$ $0.097 \pm 0.012, \mathrm{DM} 0.092 \pm 0.012, \mathrm{DM}-\mathrm{SNx} 0.102 \pm 0.013, \mathrm{p}=$ non-significant).

Nephrin Expression and Podocyte Number in DM-SNx Rats

Glomerular nephrin immunostaining was markedly lower in DM-SNx rats than in the other three groups (fig. 5a-e), while the number of glomerular cells positively immunostaining for the podocyte marker WT1 was similarly reduced in DM, SNx and DM-SNx rats relative to sham animals (fig. $5 \mathrm{f}-\mathrm{j}$ ).

\section{Discussion}

In the present study, we observed a marked augmentation of albumin excretion in DM$\mathrm{SNx}$ rats, far above that predicted by the additive effects of renal mass ablation and hyperglycemia in isolation. This impairment of the filtration barrier permselectivity occurred in 


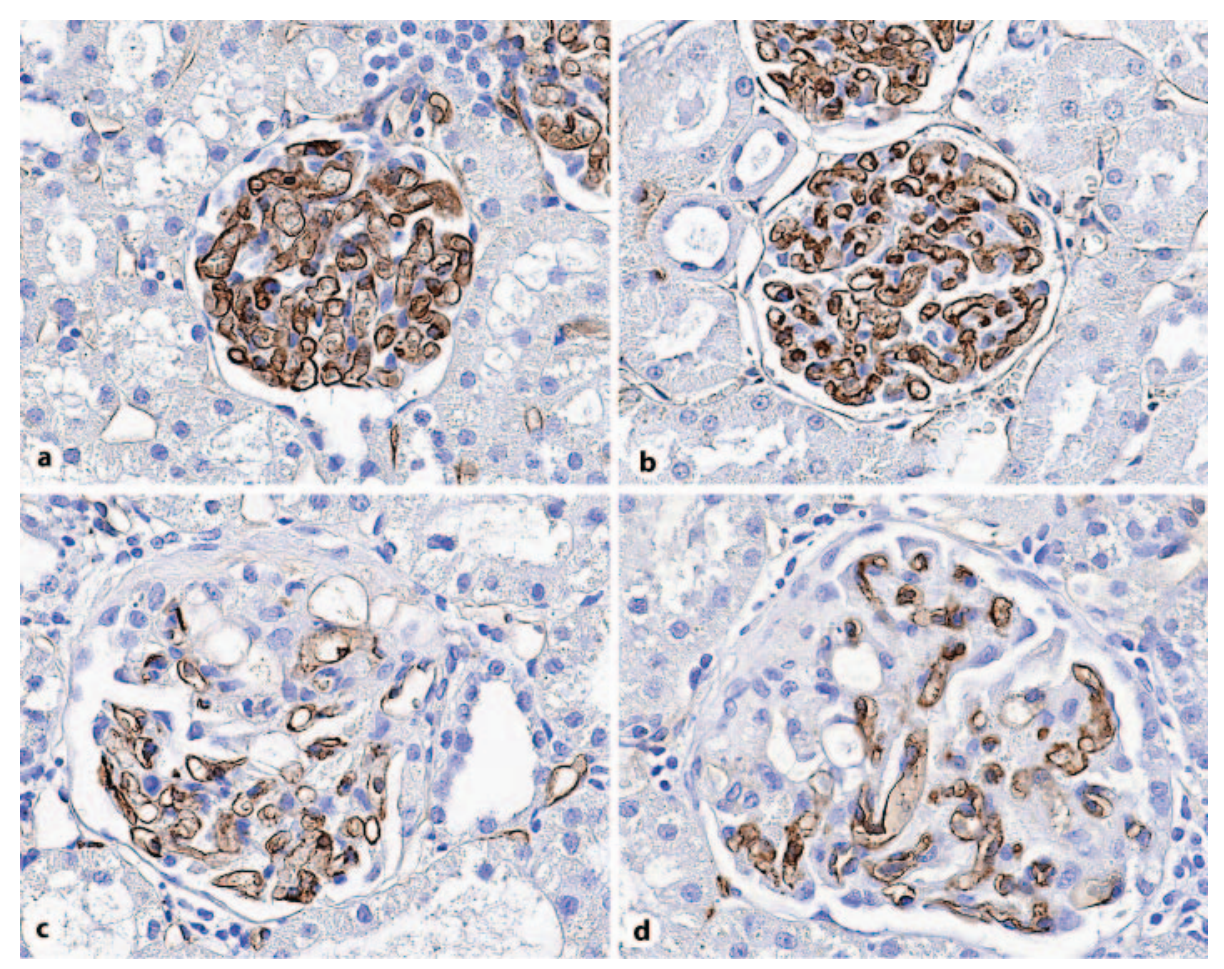

Fig. 4. Representative photomicrographs of JG-12-labeled glomerular capillaries from kidney sections from sham-operated (a), $\mathrm{DM}$ (b), $\mathrm{SNx}$ (c) and DM-SNx (d) rats. Original magnification $\times 400$. e Quantitation of JG-12 immunolabeling. ${ }^{*} \mathrm{p}<0.01$ vs. sham, ${ }^{\dagger} \mathrm{p}<0.001$ vs. DM, ${ }^{\ddagger} \mathrm{p}<$ 0.001 vs. sham.

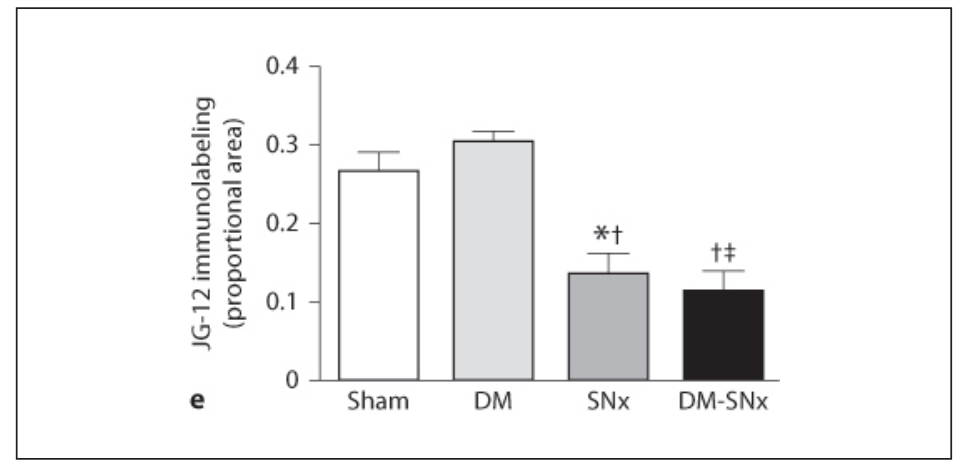

concert with augmented glomerulomegaly and a reduction in the expression of the slit pore protein nephrin, reflecting the combined effects of increased intraglomerular pressures and hyperglycemic injury. Such accelerated renal injury may be a useful means for modeling some aspects of nephropathy seen in patients.

The SNx rat is a well-characterized model of progressive proteinuric renal disease mediated by the compensatory response to rising intraglomerular pressures within the remnant kidney [12]. In contrast, hyperglycemia alone typically results in only minimal or mild renal injury in most rodent strains [1]. Surprisingly, despite their widespread adoption in isolation, few studies have examined the combined effects of STZ-induced diabetes and SNx, most likely due to the risk of peri-operative complications in rodents with marked hyperglycemia. The renal injury we observed in DM-SNx rats is comparable with that induced by two-step removal of 3/4 of the kidney mass before STZ injection [13-15], while circumventing the administration of STZ to a renally insufficient animal.

As with patients with diabetic nephropathy [16], the $\mathrm{SNx}$ rat demonstrates sensitivity to renin-angiotensin system (RAS) blockade exceeding that expected from blood pressure low- 


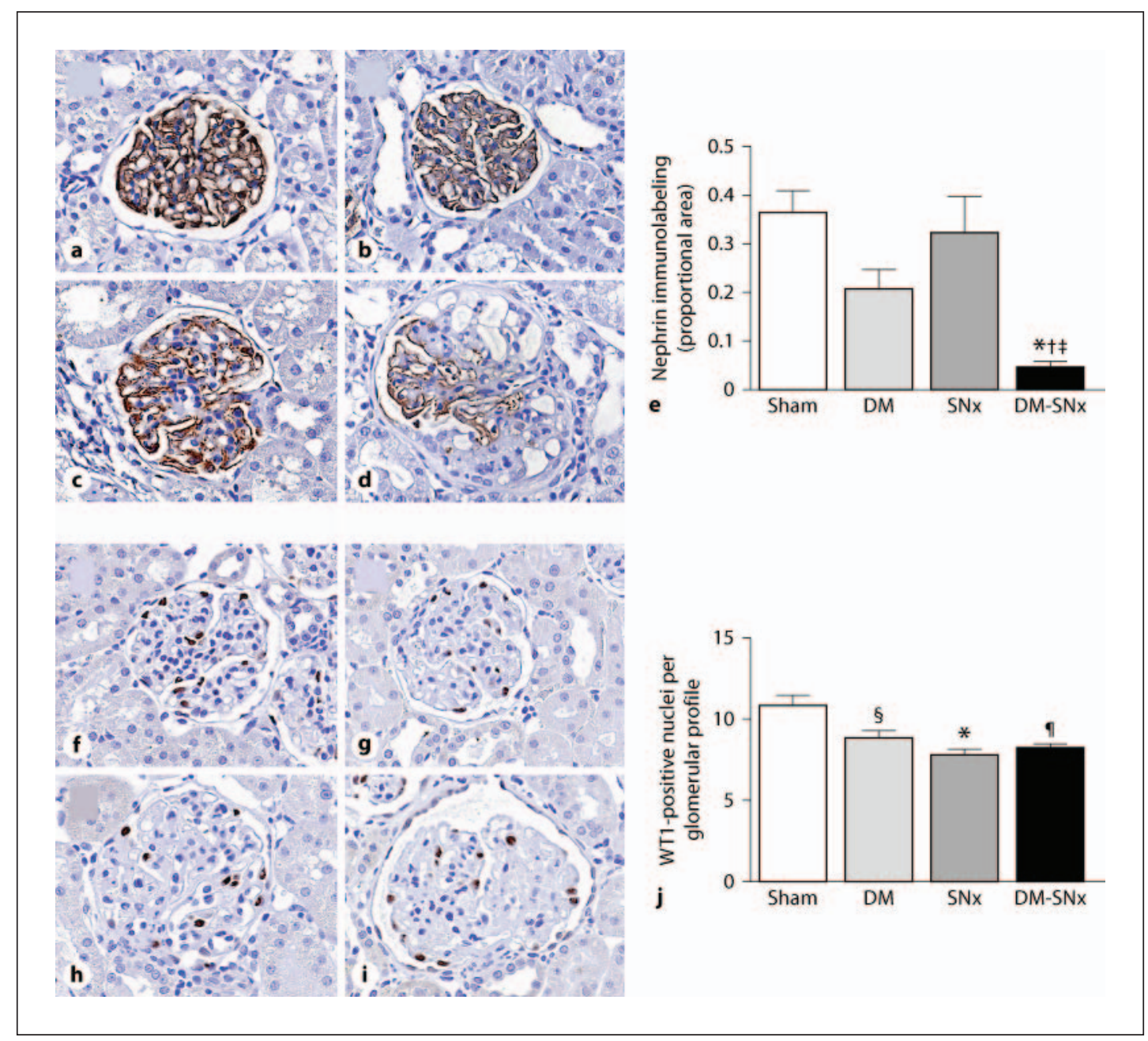

Fig. 5. Podocyte changes in DM-SNx rats. Representative photomicrographs of nephrin-immunostained kidney sections from sham-operated (a), DM (b), SNx (c) and DM-SNx (d) rats. Original magnification $\times 400$. e Quantitation of nephrin immunostaining. Representative photomicrographs of WT1-immunostained kidney sections from sham-operated (f), DM (g), $\mathrm{SNx}(\mathbf{h})$ and DM-SNx (i) rats. Original magnification $\times 400$. $\mathbf{j}$ Quantitation of WT1 immunostaining. ${ }^{*} \mathrm{p}<0.001 \mathrm{vs}$. sham, ${ }^{\dagger} \mathrm{p}<0.05 \mathrm{vs}$. DM, ${ }^{\ddagger} \mathrm{p}<0.01$ vs. $\mathrm{SNx},{ }^{\S} \mathrm{p}<0.05$ vs. sham, ${ }^{\natural} \mathrm{p}<0.01$ vs. sham.

ering alone [17]. Intriguingly, the augmented albuminuria we observed with the combination of hyperglycemia and renal mass ablation is remarkably consistent with that previously reported in an alternative hypertensive model that combined STZ-induced diabetes with angiotensin II infusion [18]. Whether the renal injury in DM-SNx rats is sensitive to conventional therapy with RAS blockade, or novel interventions, remains to be determined. One likely contributor to the heavy albuminuria in DM-SNx rats is the downregulation of the slit pore protein nephrin, which occurred independently of a decrease in podocyte number (WT1 immunostaining). While damage to any of the three principal layers of the glomerular filtration barrier may result in macromolecular leakage into the urinary space, over recent years attention has turned to a central role for podocyte injury [19]. Nephrin plays a pivotal role in maintaining the podocyte slit diaphragm [20,21], and a number of reports have dem- 
onstrated reduced nephrin expression in both experimental diabetes and after $\mathrm{SNx}$ as well as in their clinical correlates [22-25].

By employing a differential sieving approach, Kim et al. [26] demonstrated that nephrin expression was specifically reduced in hypertrophied glomeruli of diabetic rats, but not in smaller-sized glomeruli. Early glomerular enlargement is a feature of both nephron mass reduction and experimental diabetes, although the pathogenetic processes activated by these two experimental approaches are likely to be different [27]. In the present study, the glomerular volume was significantly greater in DM-SNx rats than in the other three groups, suggesting that enhanced glomerular enlargement may contribute to the downregulation of nephrin and augmented albuminuria in these animals. However, while this represents one pathogenetic process it is unlikely to be the sole one, given the magnitude of increase in albumin excretion observed in these animals.

Histologically, excessive accumulation of the extracellular matrix within the glomerular mesangium correlates most closely with GFR decline in advancing renal disease [28]. In the present study, the magnitude of glomerulosclerosis in DM and SNx rats was equivalent, although its pattern of distribution was qualitatively quite different, with a diffuse increase in mesangial matrix deposition occurring in the former and focal glomerular injury a feature of the latter. While DM-SNx rats displayed an increase in the proportion of severely sclerosed glomeruli, the absence of accelerated GFR decline in these animals is indicative of the hyperfiltrative effects of hyperglycemia alone.

Despite heavy albuminuria and augmented renal injury, like all currently available models of nephropathy the DM-SNx rat is not without its limitations. For instance, pathological changes were most prominent within the glomeruli. In contrast, tubulointerstitial fibrosis, that also correlates with advancing renal injury [29], was relatively unaffected. A further methodological limitation is the limited tissue available (1/6 of the renal mass) for subsequent molecular biological and/or histological analysis. The mechanism by which renal injury was induced may also be argued as not being representative of the clinical setting. However, this is no more so than in models that rely on the mutation, deletion or overexpression of genes implicated in the pathogenetic process. Hemodynamic forces play a fundamental role in the pathogenesis of diabetic nephropathy and, in this respect, the DM-SNx rat may be viewed as offering advantages in modeling some aspects of diabetic nephropathy including the presence of concurrent hypertension.

In summary, the DM-SNx rat model may be considered a complementary addition to the inventory of tools now available for the study of pathogenetic processes and novel therapies in diabetic nephropathy.

\section{Acknowledgements}

The authors thank Ms. Bridgit Bowskill for her excellent technical assistance. These studies were supported by CIHR grant MOP-97791. Mr. Li-Hao Chen is a recipient of a CIHR Frederick Banting and Charles Best Graduate Scholarship and a Banting and Best Diabetes Centre Graduate Studentship. Dr. Andrew Advani is a Canadian Diabetes Association Clinician Scientist, Dr. Gilbert is a Canada Research Chair in Diabetes Complications and this work was supported thanks, in part, to the Canadian Diabetes Association, the Canada Research Chair Program and a CIHR team grant in diabetes complications. Dr. Connelly is supported by a Clinician Scientist Award from the Heart and Stroke Foundation of Ontario. Dr. Yuen was previously supported by a KRESCENT postdoctoral fellowship and is currently the recipient of a Canadian Society of Transplantation postdoctoral fellowship. 


\section{References}

-1 Brosius FC 3rd, Alpers CE, Bottinger EP, Breyer MD, Coffman TM, Gurley SB, Harris RC, Kakoki M, Kretzler M, Leiter EH, Levi M, McIndoe RA, Sharma K, Smithies O, Susztak K, Takahashi N, Takahashi T: Mouse models of diabetic nephropathy. J Am Soc Nephrol 2009;20:2503-2512.

-2 Advani A, Huang Q, Thai K, Advani SL, White KE, Kelly DJ, Yuen DA, Connelly KA, Marsden PA, Gilbert RE: Long-term administration of the histone deacetylase inhibitor vorinostat attenuates renal injury in experimental diabetes through an endothelial nitric oxide synthase-dependent mechanism. Am J Pathol 2011;178:2205-2214.

-3 Klein R, Klein BE, Lee KE, Cruickshanks KJ, Moss SE: The incidence of hypertension in insulindependent diabetes. Arch Intern Med 1996;156:622-627.

-4 Ueda H, Ishimura E, Shoji T, Emoto M, Morioka T, Matsumoto N, Fukumoto S, Miki T, Inaba M, Nishizawa Y: Factors affecting progression of renal failure in patients with type 2 diabetes. Diabetes Care 2003;26:1530-1534.

-5 Brenner BM, Cooper ME, de Zeeuw D, Keane WF, Mitch WE, Parving HH, Remuzzi G, Snapinn SM, Zhang Z, Shahinfar S: Effects of losartan on renal and cardiovascular outcomes in patients with type 2 diabetes and nephropathy. N Engl J Med 2001;345:861-869.

-6 Lewis EJ, Hunsicker LG, Bain RP, Rohde RD: The effect of angiotensin-converting-enzyme inhibition on diabetic nephropathy. The Collaborative Study Group. N Engl J Med 1993;329:1456-1462.

-7 Meyer TW, Anderson S, Rennke HG, Brenner BM: Converting enzyme inhibitor therapy limits progressive glomerular injury in rats with renal insufficiency. Am J Med 1985;79:31-36.

-8 Advani A, Connelly KA, Yuen DA, Zhang Y, Advani SL, Trogadis J, Kabir G, Shachar E, Kuliszewski MA, Leong-Poi H, Stewart DJ, Gilbert RE: Fluorescent microangiography is a novel and widely applicable technique for delineating the renal microvasculature. PLoS One 2011;6:e24695.

-9 Advani A, Kelly DJ, Advani SL, Cox AJ, Thai K, Zhang Y, White KE, Gow RM, Marshall SM, Steer BM, Marsden PA, Rakoczy PE, Gilbert RE: Role of VEGF in maintaining renal structure and function under normotensive and hypertensive conditions. Proc Natl Acad Sci USA 2007;104:1444814453.

-10 Advani A, Wiggins KJ, Cox AJ, Zhang Y, Gilbert RE, Kelly DJ: Inhibition of the epidermal growth factor receptor preserves podocytes and attenuates albuminuria in experimental diabetic nephropathy. Nephrology (Carlton) 2011;16:573-581.

-11 Gilbert RE, Huang Q, Thai K, Advani SL, Lee K, Yuen DA, Connelly KA, Advani A: Histone deacetylase inhibition attenuates diabetes-associated kidney growth: potential role for epigenetic modification of the epidermal growth factor receptor. Kidney Int 2011;79:1312-1321.

-12 Blantz RC, Gabbai FB: Glomerular hemodynamics in pathophysiologic conditions. Am J Hypertens 1989;2:208S-212S.

13 Elrashidy RA, Asker ME, Mohamed HE: Pioglitazone attenuates cardiac fibrosis and hypertrophy in a rat model of diabetic nephropathy. J Cardiovasc Pharmacol Ther 2012, E-pub ahead of print.

-14 Yamabe N, Yokozawa T, Oya T, Kim M: Therapeutic potential of (-)-epigallocatechin 3-O-gallate on renal damage in diabetic nephropathy model rats. J Pharmacol Exp Ther 2006;319:228-236.

-15 Yokozawa T, Nakagawa T, Wakaki K, Koizumi F: Animal model of diabetic nephropathy. Exp Toxicol Pathol 2001;53:359-363.

16 Ruggenenti P, Cravedi P, Remuzzi G: The RAAS in the pathogenesis and treatment of diabetic nephropathy. Nat Rev Nephrol 2010;6:319-330.

-17 Jackson B, Johnston CI: Angiotensin-converting enzyme inhibition in renal disease; contrasting effects on renal function in renal artery stenosis and progressive renal injury. J Hum Hypertens 1989; 3(suppl 1):107-115.

-18 Nicholas SB, Mauer M, Basgen JM, Aguiniga E, Chon Y: Effect of angiotensin II on glomerular structure in streptozotocin-induced diabetic rats. Am J Nephrol 2004;24:549-556.

19 Wolf G, Chen S, Ziyadeh FN: From the periphery of the glomerular capillary wall toward the center of disease: podocyte injury comes of age in diabetic nephropathy. Diabetes 2005;54:1626-1634.

-20 Holzman LB, St John PL, Kovari IA, Verma R, Holthofer H, Abrahamson DR: Nephrin localizes to the slit pore of the glomerular epithelial cell. Kidney Int 1999;56:1481-1491.

-21 Ruotsalainen V, Ljungberg P, Wartiovaara J, Lenkkeri U, Kestila M, Jalanko H, Holmberg C, Tryggvason K: Nephrin is specifically located at the slit diaphragm of glomerular podocytes. Proc Natl Acad Sci USA 1999;96:7962-7967. 
-22 Langham RG, Kelly DJ, Cox AJ, Thomson NM, Holthofer H, Zaoui P, Pinel N, Cordonnier DJ, Gilbert RE: Proteinuria and the expression of the podocyte slit diaphragm protein, nephrin, in diabetic nephropathy: effects of angiotensin converting enzyme inhibition. Diabetologia 2002;45:1572-1576.

-23 Kelly DJ, Edgley AJ, Zhang Y, Thai K, Tan SM, Cox AJ, Advani A, Connelly KA, Whiteside CI, Gilbert RE: Protein kinase C-beta inhibition attenuates the progression of nephropathy in non-diabetic kidney disease. Nephrol Dial Transplant 2009;24:1782-1790.

- 24 Kelly DJ, Aaltonen P, Cox AJ, Rumble JR, Langham R, Panagiotopoulos S, Jerums G, Holthofer H, Gilbert RE: Expression of the slit-diaphragm protein, nephrin, in experimental diabetic nephropathy: differing effects of anti-proteinuric therapies. Nephrol Dial Transplant 2002;17:1327-1332.

25 Bennett MR, Czech KA, Arend LJ, Witte DP, Devarajan P, Potter SS: Laser capture microdissectionmicroarray analysis of focal segmental glomerulosclerosis glomeruli. Nephron 2007;107:e30-e40.

-26 Kim JJ, Li JJ, Jung DS, Kwak SJ, Ryu DR, Yoo TH, Han SH, Choi HY, Kim HJ, Han DS, Kang SW: Differential expression of nephrin according to glomerular size in early diabetic kidney disease. J Am Soc Nephrol 2007;18:2303-2310.

-27 Seyer-Hansen K: Renal hypertrophy in experimental diabetes mellitus. Kidney Int 1983;23:643-646.

-28 Mauer SM, Lane P, Hattori M, Fioretto P, Steffes MW: Renal structure and function in insulin-dependent diabetes mellitus and type I membranoproliferative glomerulonephritis in humans. J Am Soc Nephrol 1992;2:S181-S184.

-29 Gilbert RE, Cooper ME: The tubulointerstitium in progressive diabetic kidney disease: more than an aftermath of glomerular injury? Kidney Int 1999;56:1627-1637. 